\title{
Los nombres de pila en Alemania como portadores de información social. ¿Cómo se puede codificar la información social en los nombres?
}

\section{First names in Germany as carriers of social information.How can social information be encoded in names?}

\section{Resumen}

Además de la función de identificación de su portador, los nombres de pila están asociados a mucha información sobre la persona y su entorno social porque la elección del nombre está guiada por las experiencias de vida de los padres, inmersos en un contexto social. La elección es influida por circunstancias regionales y temporales, tradiciones familiares, modelos de conducta, religión, pertenencia a grupos sociales $\mathrm{y}$, hoy más que nunca, por aspectos estéticos. Por lo tanto, un nombre de pila siempre lleva informaciones sociales sobre el género, la edad o la afiliación a un grupo social. Dentro de una cultura y dentro de una época, esta información se percibe de manera similar. Fürchtegott 'temo de Dios', Gottlieb 'amor a Dios' se perciben como nombres pietistas; Mandy, Peggy, Enrico como nombres típicos de la República Democrática Alemana (RDA); Adolf y Horst como nombres del tiempo del nazismo; Ali, Mohamed y Aishe como nombres extranjeros. ¿Qué pasa con las otras características? ¿Hay más connotaciones globales y cómo se pueden hacer visibles? Se presenta la investigación "The Image of Names" (la imagen de los nombres de pila), sobre la percepción de estos nombres, cuyo núcleo es una encuesta en línea. La encuesta capta el efecto individual de una serie de nombres con respecto a pares de connotación situados en un diferencial semántico. Las parejas de connotaciones se relacionan con el sentido del nombre en sí mismo, así como con las atribuciones a los respectivos supuestos portadores del nombre. Entre otras cosas, se explora la percepción de la eufonía, la religiosidad, la apariencia y el estatus social. Ya en los años 1970 y 1980, hubo estudios en Alemania que indicaban que ciertos rasgos de carácter también se percibían en todos los ámbitos. El corpus de nombres aquí analizado es mayor (más de 2000 nombres) y se recopilan grandes cantidades de datos (150 000 cuestionarios en noviembre de 2016). A partir de los datos recopilados, se calculan los perfiles de impacto gráfico, los llamados onogramas, que muestran si y en qué áreas los nombres tienen connotaciones con efecto general dentro del mundo de habla alemana. Los resultados se pueden comparar con los datos estadísticos de los nombres de pila.

Palabras clave: Socioantroponimia; percepción de los nombres de pila; onogramas, Alemania, siglos XX y XXI. 
Onomástica Desde América Latina, n.1, v.1, janeiro - junho, 2020, p. 77 -99. ISSN 2675-2719

https://doi.org/10.48075/odal.v1i1.24161

\begin{abstract}
In addition to the identification function carried by first names, first names are associated with a lot of information about the person and their social environment because the choice of name is guided by the life experiences of the parents, immersed in a social context. The choice is influenced by regional and temporal circumstances, family traditions, models of conduct, religion, membership of social groups and, today more than ever, by aesthetic aspects. Therefore, a first name always carries social information about gender, age or affiliation to a social group. Within a culture and within an era, this information is perceived in a similar way. 'I fear of God', Gottlieb 'love of God' are perceived as pietist names; Mandy, Peggy, Enrico as typical names of the German Democratic Republic (GDR); Adolf and Horst as names of the time of Nazism; Ali, Mohamed and Aishe as foreign names. What about the other features? "The Image of Names" research is presented on the perception of these names, the core of which is an online survey. The survey captures the individual effect of a series of names with respect to connotation pairs located in a semantic differential. The pairs of connotations relate to the meaning of the name itself, as well as to the attributions to the respective alleged bearers of the name. Among other things, it explores the perception of euphony, religiosity, appearance and social status. As early as the 1970s and 1980s, studies were in Germany indicating that certain character traits were also perceived in all areas. The corpus of names discussed here is higher (more than 2000 names) and large amounts of data are collected (150,000 questionnaires in November 2016). From the collected data, graphical impact profiles, so-called onograms, are calculated,showing whether and in which areas the names have connotations with general effect within the German-speaking world. The results can be compared with the statistical data of the first names.
\end{abstract}

Keywords: Socioanthropymy; perception of first names; onograms, Germany, 20th and 21 st centuries.

Además de la función de identificación de su portador, los nombres de pila se asocian con mucha información sobre la persona y su entorno social. La elección del nombre de pila está guiada actualmente por las experiencias de vida de los padres, dentro de un contexto social. Dicha elección está influenciada por circunstancias regionales y temporales, tradiciones familiares, modelos de conducta, religión, pertenencia a determinados grupos sociales y, hoy más que nunca, por aspectos estéticos. Por lo tanto, un nombre de pila siempre vehicula información social y cultural, como el género, la edad o la afiliación a un grupo social. Dentro de una comunidad sociolingüística, una cultura y una época, esta información puede percibirse de manera similar. 
Onomástica Desde América Latina, n.1, v.1, janeiro - junho, 2020, p. 77 -99. ISSN 2675-2719 https://doi.org/10.48075/odal.v1i1.24161

Fürchtegott 'temor de Dios', Gottlieb 'amor a Dios' se perciben actualmente en Alemania como nombres pietistas; Mandy, Peggy y Enrico como nombres típicos de la República Federal Alemana (RDA); Adolf y Horst como nombres en el tiempo del nazismo; Ali, Mohamed y Aishe como nombres extranjeros. ¿Qué ocurre con otras connotaciones? ¿Hay más asociaciones compartidas y cómo pueden hacerse visibles? En el presente trabajo, se muestran algunos resultados obtenidos en la investigación "The Image of Names" (La imagen de los nombres de pila), la cual se ocupa sobre del efecto asociativo que ciertos nombres de pila causan en quien entra en contacto con ellos. En este estudio, se conformó un cuerpo de datos a partir de una encuesta aplicada en línea.

La encuesta pretende medir el efecto individual de ciertos nombres con respecto a pares de connotación situados sobre un diferencial semántico. Las parejas de connotaciones se relacionan con el sentido del nombre en sí mismo, así como con los atributos o características que suelen relacionarse con los respectivos supuestos portadores del nombre.

Entre otras cosas, se consideraron para los cuestionarios las percepciones sobre la eufonía, la religiosidad, la apariencia y el estatus social. Ya en los años 1970 y 1980, hubo estudios en Alemania que indicaban claramente que ciertos rasgos de carácter también eran atribuidos a los portadores de algunos nombres, como percepciones generales en todos los ámbitos. ${ }^{1}$

A diferencia de los trabajos de Krien (1973) y de Hartmann (1984), el corpus de nombres aquí analizado es mayor (más de 2.000 nombres) y se recopilaron grandes

\footnotetext{
${ }^{1}$ HARTMANN T. (1984) Untersuchung der konnotativen Bedeutung von Personennamen. Ein theoretischer und empirischer Beitrag zur Psychoonomastik mit Hilfe eines konzeptspezifischen semantischen Differentials. Neumünster: Wachholz; KRIEN R. (1973) Namenphysiognomik: Untersuchungen zur sprachlichen Expressivität am Beispiel von Personennamen, Appellativen und Phonemen des Deutschen. Tübingen: Max Niemeyer Verlag.
} 
Onomástica Desde América Latina, n.1, v.1, janeiro - junho, 2020, p. 77 -99. ISSN 2675-2719 https://doi.org/10.48075/odal.v1i1.24161

cantidades de datos (150.000 "votos" — cualidades atribuidas a los portadores de un mismo nombre- hasta noviembre de 2016). A partir de los datos colectados, se calculan los perfiles de impacto gráfico, los llamados onogramas, que muestran si y en qué áreas los nombres evocan connotaciones generalizadas dentro del mundo de habla alemana. Estos se pueden comparar con los datos estadísticos de los nombres de pila y mostrar los cambios a largo plazo en la percepción que se tiene sobre dichos nombres.

Para esto, los nombres se evalúan de acuerdo con trece criterios, que luego muestran si el nombre del portador tiende a asociarse más con ciertas propiedades (pares de connotación) o con otras. Es importante aclarar que las evaluaciones, por supuesto, no se aplican a portadores individuales de nombres, sino que reflejan la idea que la mayoría de las personas tiene al escuchar el nombre.

Estos perfiles de impacto son el resultado de una encuesta en línea continua y, por lo tanto, cambian con el tiempo. Los onogramas actuales muestran un extracto desde 2014 hasta agosto de 2017. Los resultados actuales se pueden encontrar en la página web: www.onomastik.com. Este trabajo pertenece a una investigación más amplia sobre nombres de pila en Alemania.

A continuación, se explica brevemente la manera de leer los onogramas. En las primeras tres columnas se pregunta al encuestado cómo percibe determinado nombre de pila, cuando lo escucha: muy familiar o extraño, de moda o anticuado y eufónico o no eufónico.

Después, se solicita al encuestado que caracterice a una persona con ese nombre: ¿es ella hombre o mujer, joven o mayor, simpática o antipática, antideportiva o deportiva, 
Onomástica Desde América Latina, n.1, v.1, janeiro - junho, 2020, p. 77 -99. ISSN 2675-2719 https://doi.org/10.48075/odal.v1i1.24161

tranquila o enérgica, sociable o solitaria, pobre o rica, atractiva o no atractiva, religiosa o atea e inteligente o no inteligente? (ver Figura 1).

Percibo el nombre Thilo como... (1 muy familiar o extraño, 2 de moda o anticuado, 3 eufónico o no eufónico)

Creo que quien se llama Thilo es... (4 hombre o mujer, 5 joven o mayor, 6 simpática o antipática, 7 antideportiva o deportiva, 8 tranquila o enérgica, 9 sociable o solitaria, 10 pobre o rica, 11 atractiva o no atractiva, 12 religiosa o atea, 13 inteligente o no inteligente)

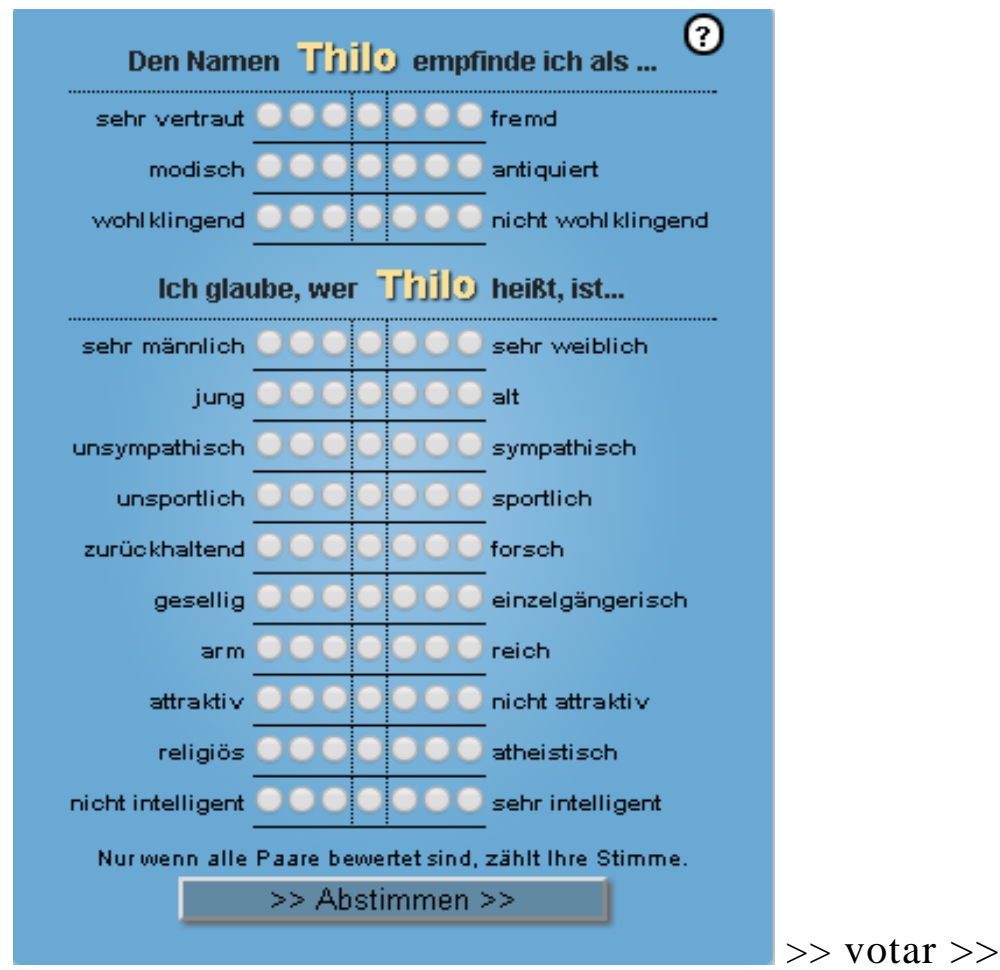

Figura 1: Cuestionario en línea para la percepción de los nombres de pila (www.onomastik.com)

Las barras indican cómo los encuestados calificaron un nombre. Si un nombre es discreto con respecto a un par de connotaciones, la media de ese par muestra la erupción más alta. Muchos nombres, especialmente los nombres usuales y de larga tradición en Alemania, como Stefan, Claudia o Gabriele, muestran pequeñas dispersiones. Eso no es raro. Cuanto más a la izquierda o a la derecha del centro se concentran los valores, más claramente está el nombre asociado a una connotación general. 
Onomástica Desde América Latina, n.1, v.1, janeiro - junho, 2020, p. 77 -99. ISSN 2675-2719 https://doi.org/10.48075/odal.v1i1.24161

Así, por ejemplo, Gottlieb evoca la imagen de un hombre muy religioso y Willy, una persona muy sociable. Incluso las posiciones en la escala se pueden interpretar: un Frank parece más masculino que un Jens y una Maria parece más atractiva que una Ute. Los valores pueden acumularse tanto a la izquierda como a la derecha, como la percepción de la edad del nombre de pila Greta. Esto refleja que este nombre está experimentando un renacimiento en el gusto de los alemanes. Es decir, está iniciando un ciclo de moda.

A modo de comparación, cómo parte de este trabajo, se evaluaron los nombres de pila más populares de los últimos 100 años en Alemania.

Los nombres masculinos más frecuentes a principios del siglo XX fueron los siguientes: Alfred, Art(h)ur, Adolf/Adolph, Bruno, Erich, Ernst, Franz, Eberhard, Friedrich, Fritz, Georg, Gerhard, Günt(h)er, Heinrich, Heinz, Helmut(h), Herman(n), Horst, Joachim, Karl/Carl, Karlheinz/Karl-Heinz, Klaus/Claus, Kurt/Curt, Manfred, Max, Otto, Paul, Richard, Rudolf/Rudolph, Walt(h)er, Werner, Wilhelm, Willi/Willy, Wolfgang. Los nombres femeninos más frecuentes: Anna/Anne/Anni/Anneliese, Bärbel, Bert(h)a, Elfriede, Elisabeth, Elsa/Else, Erika, Erna, Fri(e)da, Gertrud, Gisela, Hedwig, Helga, Hert(h)a, Ida, Ingeborg, Irmgard, Kät(h)e, Lieselotte, Margaret(h)e, Maria/Marie, Ruth, Ursula, Waltraud. 
Onomástica Desde América Latina, n.1, v.1, janeiro - junho, 2020, p. 77 -99. ISSN 2675-2719 https://doi.org/10.48075/odal.v1i1.24161

Analizando los onogramas de los nombres de pila de esta época como Elfriede, Karlheinz, Arthur y Adolf (Figuras 2-5), se puede ver que las personas con estos nombres de pila tienden a ser calificados como notablemente más viejos que los que se mencionarán después. Por otro lado, las personas con el nombre de pila Adolfo, con un prestigio bastante negativo en Alemania, también se calificaban como muy antipáticas y poco atractivas.

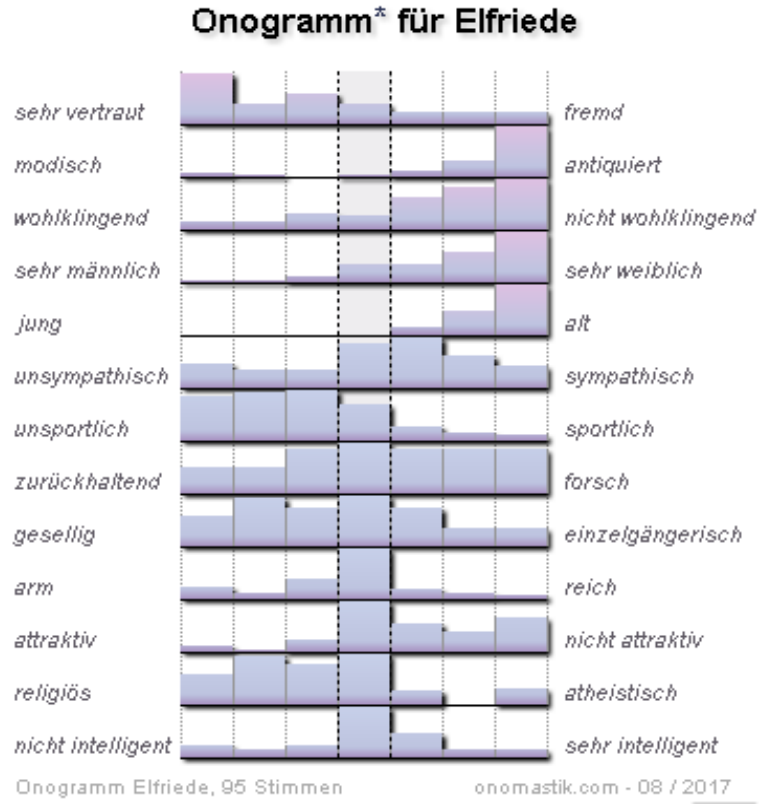

Figura 2: Elfriede

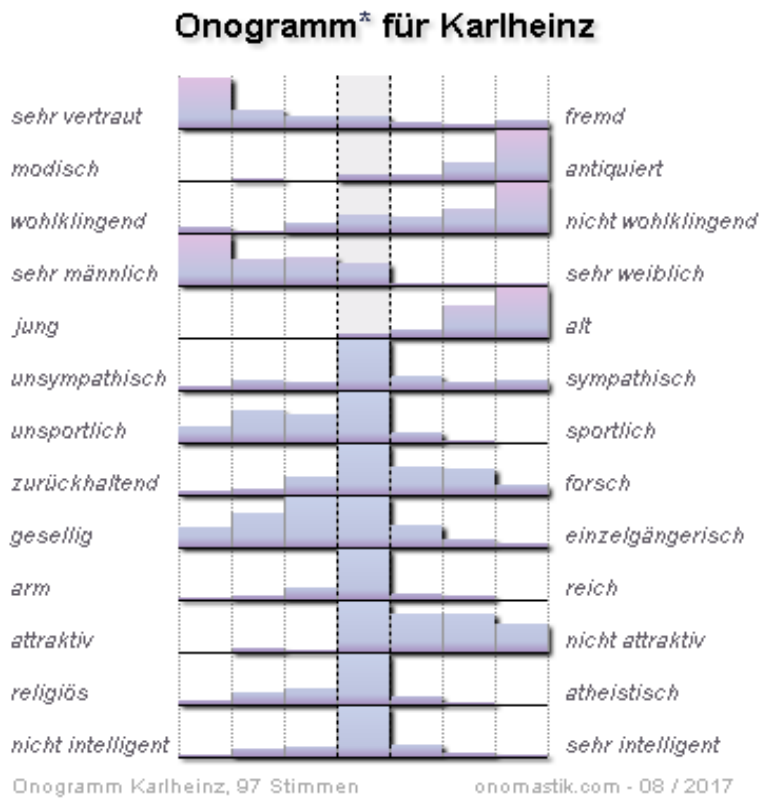

Figura 3: Karlheinz 
Onomástica Desde América Latina, n.1, v.1, janeiro - junho, 2020, p. 77 -99. ISSN 2675-2719 https://doi.org/10.48075/odal.v1i1.24161

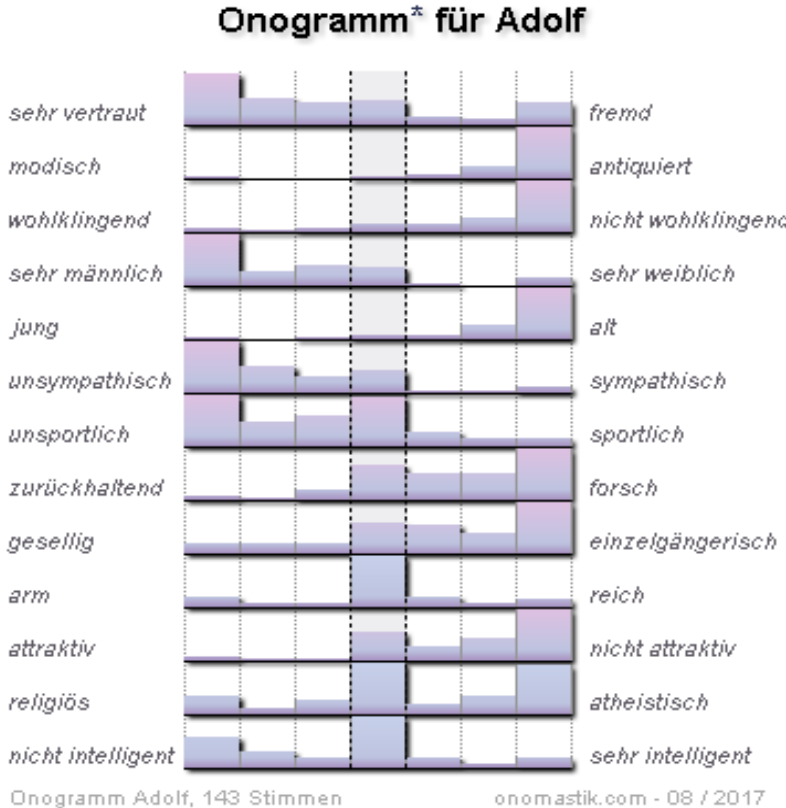

Figura 4: Arthur
Onogramm für Arthur

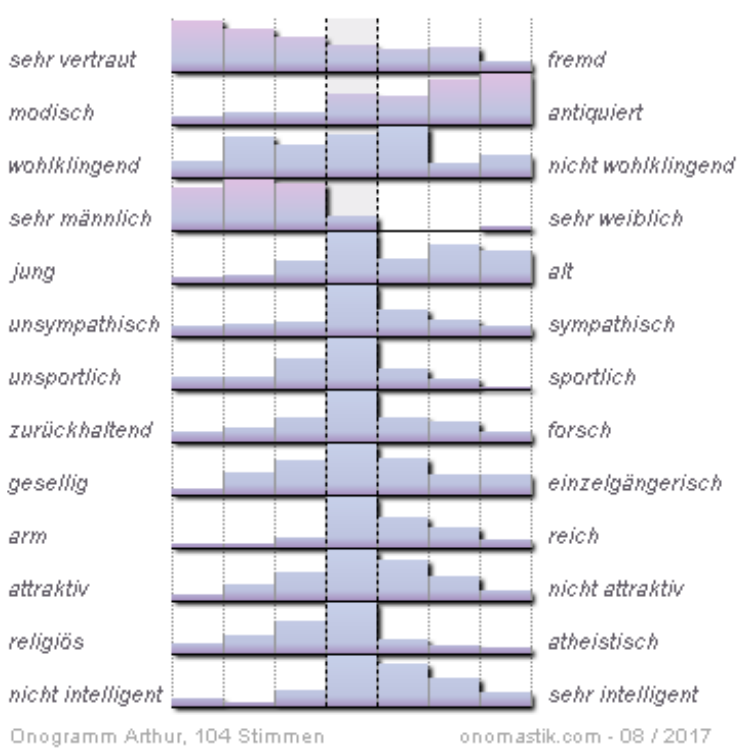

Figura 5: Adolf

Después de la Segunda Guerra Mundial cambió la manera de atribuir un nombre de pila en los países de habla alemana. Empezó la era de la libertad de elección. Los nombres femeninos más populares en la década de los años 50 fueron Angelika, Brigitte, Gabriele, Ingrid, Karin, Monika, Renate, Sabin, Ursula, Ute, y los nombres masculinos Bernd, Hans, Jürgen, Klaus/Claus, Michael, Peter, Rainer, Ralf, Thomas, Wolfgang.

Los supuestos portadores de estos nombres de pila también son percibidos como mayores, pero significativamente menos que los nombres mencionados en el punto 1 (Figuras 6-9). 
Onomástica Desde América Latina, n.1, v.1, janeiro - junho, 2020, p. 77 -99. ISSN 2675-2719 https://doi.org/10.48075/odal.v1i1.24161

\section{Onogramm ${ }^{\star}$ für Angelika}

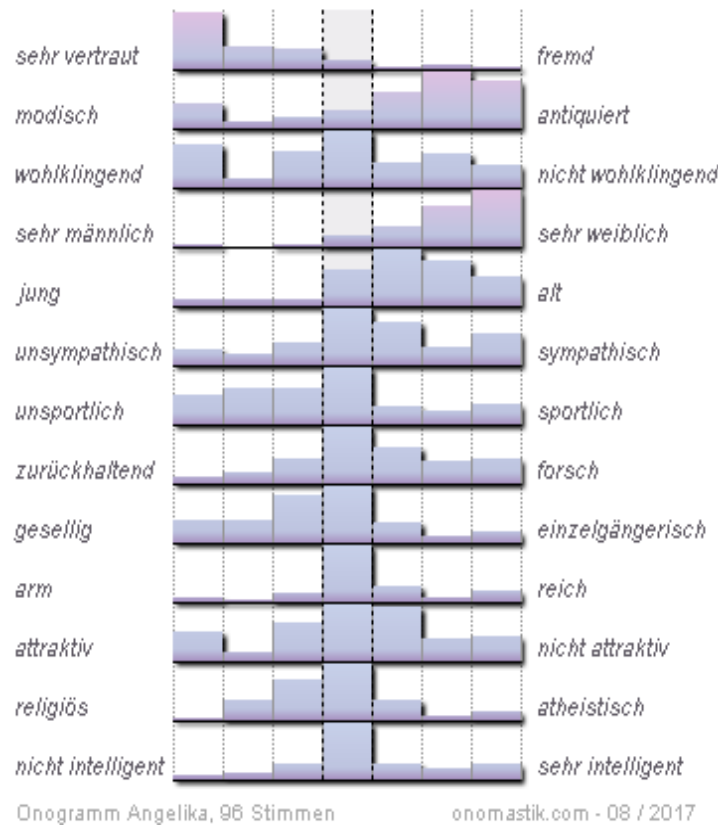

\section{Onogramm für Monika}

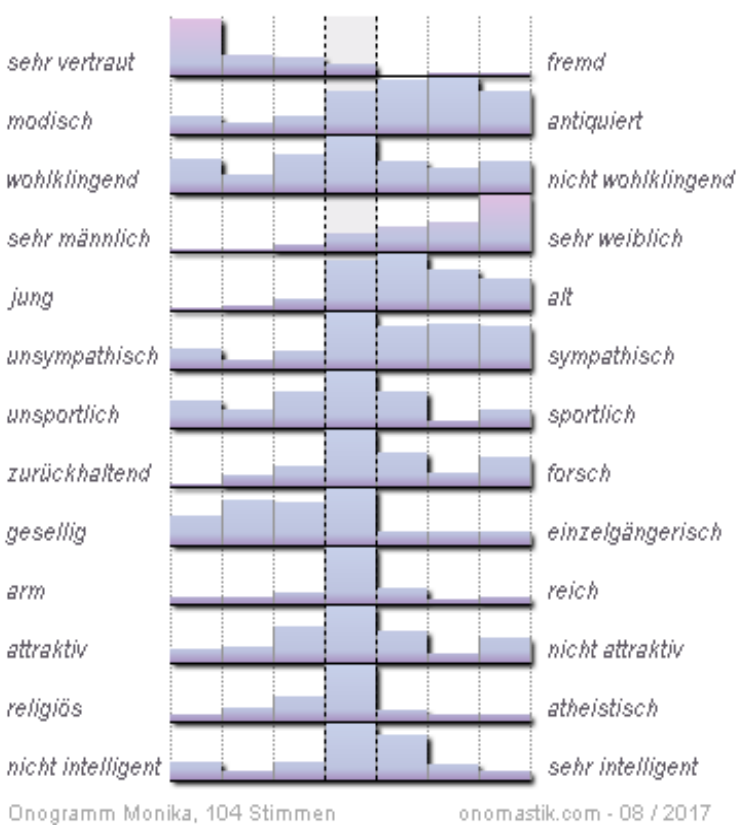

\section{Onogramm für Jürgen}

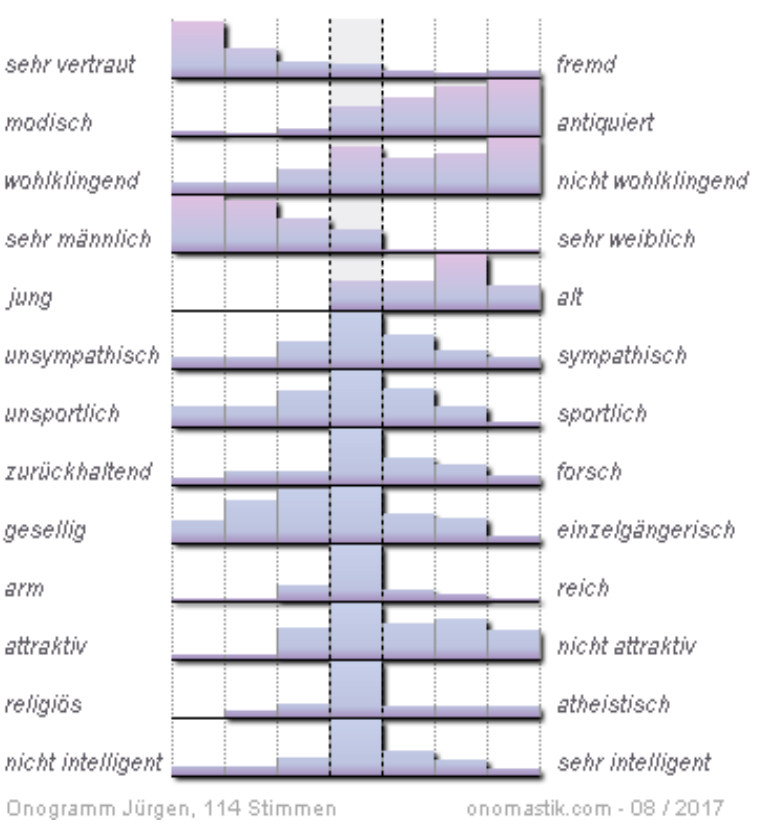

Figura 6: Angelika

Figura 8: Jürgen

\section{Onogramm für Ralf}

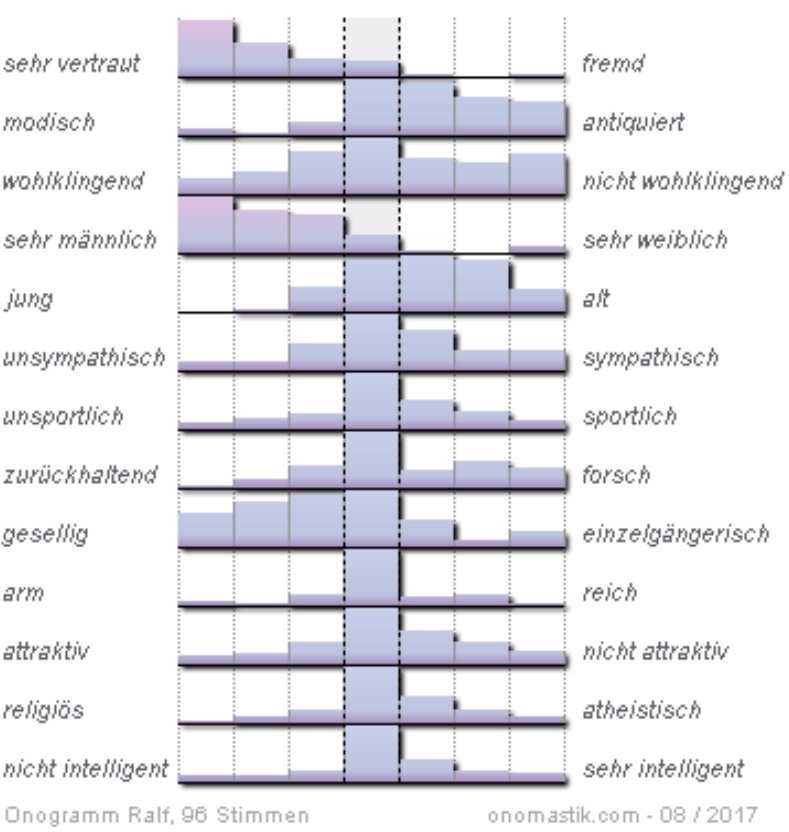

Figura 7: Monika

Figura 9: Ralf 
Onomástica Desde América Latina, n.1, v.1, janeiro - junho, 2020, p. 77 -99. ISSN 2675-2719 https://doi.org/10.48075/odal.v1i1.24161

Los nombres femeninos más populares en la década de los años 80 fueron Anna, Anne, Christine, Christina, Franziska, Julia, Katharina, Nadine, Nicole, Sabrina, Stefanie/Stephanie, y los nombres masculinos fueron Alexander, Christian, Daniel, Martin, Matthias, Michael, Patrick, Robert, Sebastian, Stefan/Stephan.

En los años 90 los nombres femeninos más populares fueron Anna, Anne, Julia, Katharina, Laura, Lisa, Sara(h), Maria, Marie, Michelle, Sophie/Sofie y los nombres masculinos Alexander, Christian, Daniel, Florian, Kevin, Lukas/Lucas, Maximilian, Michael, Paul, Philipp.

La mayoría de estos nombres de pila se puede encontrar hoy en la lista de los nombres más frecuentes entre los recién nacidos en Alemania. Estos son nombres de pila con una larga tradición. A pesar de llevar largo tiempo en uso, se perciben como nombres bellos y que suenan bien.

Estos nombres, que no son ni muy antiguos ni muy modernos, son atemporales por pertenecer al repertorio "clásico". Por ejemplo, los nombres Maria, Anna y Alexander se perciben al mismo tiempo como nombres muy conocidos, anticuados, y de moda, según los resultados numéricos del cuestionario aplicado. A diferencia de los nombres de los grupos 1 y 2, los encuestados expresan que estos nombres suenan bien y que los portadores de estos nombres de pila son bastante atractivos y simpáticos (Figuras 10-12). 
Onomástica Desde América Latina, n.1, v.1, janeiro - junho, 2020, p. 77 -99. ISSN 2675-2719 https://doi.org/10.48075/odal.v1i1.24161

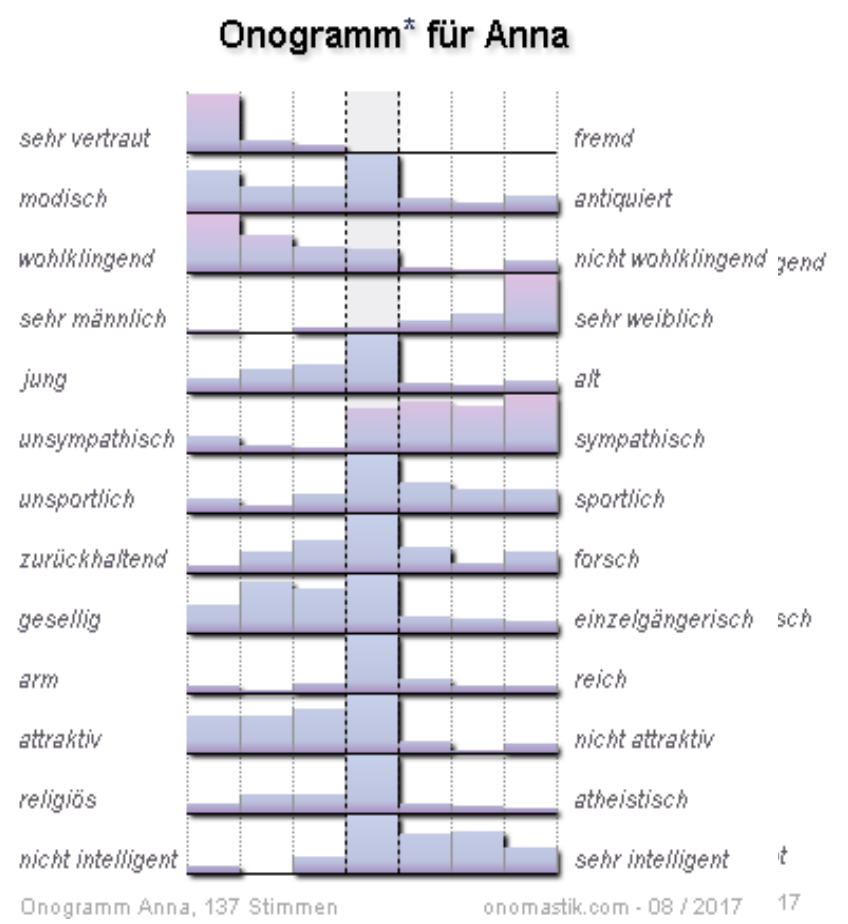

Figura 12: Anna

Los nombres de los países de habla inglesa como Kevin son muy populares en familias socialmente frágiles y de menos recursos. Numerosos estudios sobre los nombres de pila, como una tesis de maestría del Centro de investigación sobre la infancia de la Universidad de Oldenburg "Carl von Ossietzky" (Kube, 2009) informan que las personas estudiadas evalúan a los niños con los nombres Charlotte, Sophie, Marie Hana(h), Alexander, Simon, Lukas y Jakob como amables, inteligentes y sin problemas de comportamiento, mientras que a los niños con los nombres Chantal, Mandy, Angelina, Jacqueline, Kevin, Justin, Maurice, los consideran como menos inteligentes y con problemas de comportamiento. La declaración hecha por una maestra de escuela primaria: "Kevin no es un nombre, sino un diagnóstico", pasó por los medios de comunicación. Conceptos como kevinismo y chantalismo salieron a la luz, lo que dio a estos nombres una reputación negativa. En consecuencia, el nombre Kevin, que al principio de su ciclo de atribución era muy popular, ahora se considera no eufónico (ver Gráfica 14): 
Onomástica Desde América Latina, n.1, v.1, janeiro - junho, 2020, p. 77 -99. ISSN 2675-2719 https://doi.org/10.48075/odal.v1i1.24161

\section{Verlaufskurve KEVIN 1960-1990}

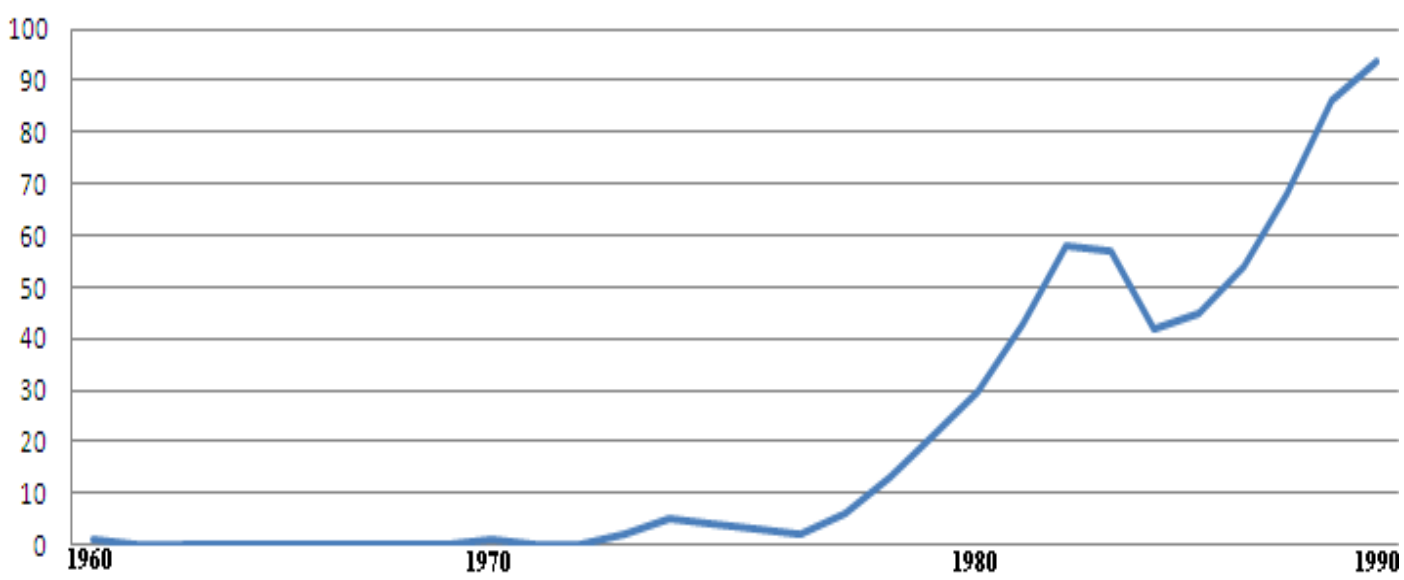

Gráfica 14: El desarrollo de los registros del nombre de pila Kevin entre 1960 y 1990 en Alemania (Base de datos del Centro de Onomástica de la Universidad de Leipzig)

Y los hombres llamados Kevin son considerados hoy día, según los resultados de nuestra encuesta, como antipáticos, pobres, poco atractivos y no inteligentes (Figura 14). Esto refleja la influencia de los medios de comunicación sobre la percepción de los

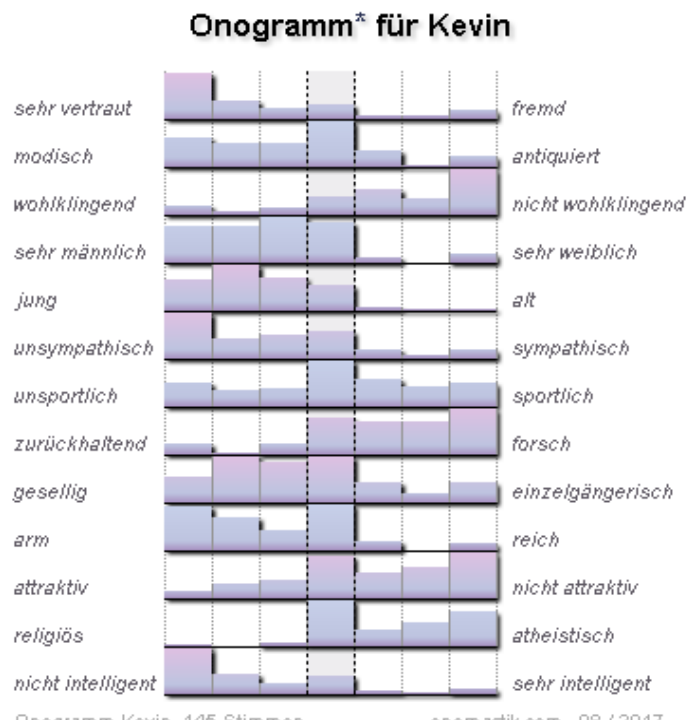

nombres, lo que aumenta los prejuicios contra los portadores del nombre Kevin.

Figura 14: Kevin 
Onomástica Desde América Latina, n.1, v.1, janeiro - junho, 2020, p. 77 -99. ISSN 2675-2719 https://doi.org/10.48075/odal.v1i1.24161

A partir del año 2000 predominan nombres de pila cortos como Mia, Lea(h), Lilli/Lilly, Anna, Anne, Emily, Emma, Hanna(h), Julia, Laura, Lena, Leni, junto a otros más largos, tales como Emilia, Johanna, Leonie, Marie, Maria, Sophie/Sofie para las niñas y Ben, May, Paul, Tim, Tom, David, Elias, Felix, Finn/Fynn, Jonas, Leon, L(o)uis, Luca/Luka, Lucas/Lukas junto a Alexander y Maximilian, para los niños. Estos nombres se perciben

\section{Onogramm für Leon}

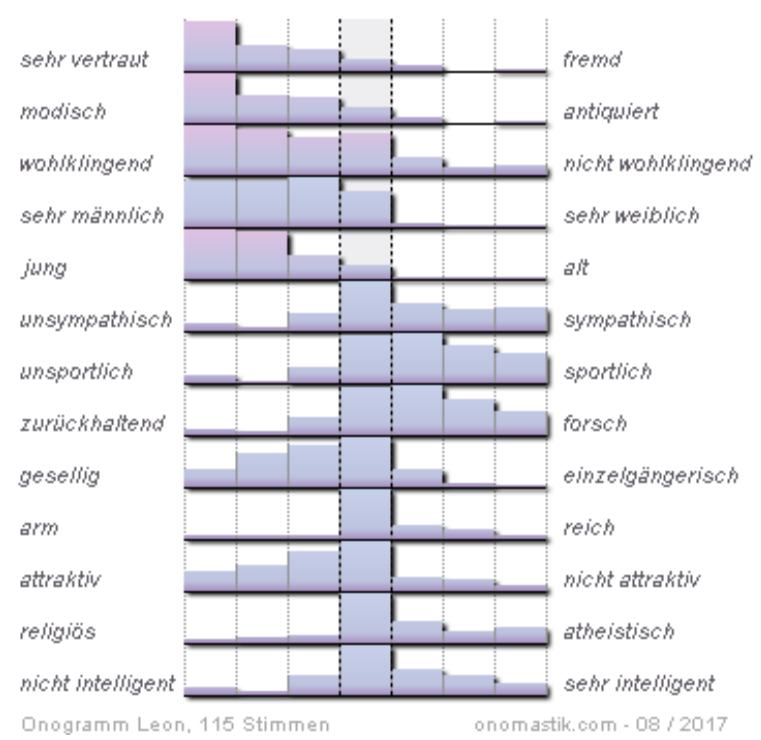

Onogramm für Finn

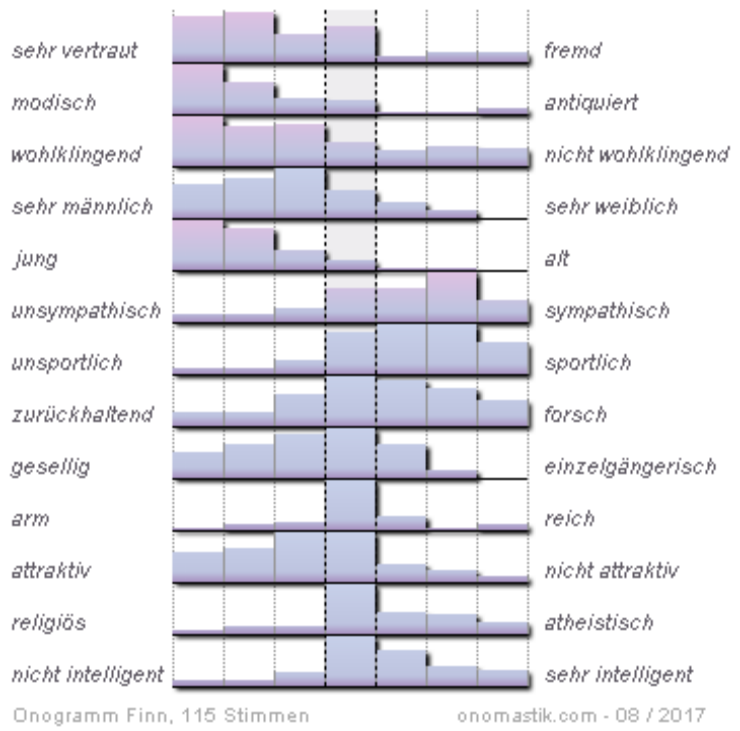

como melodiosos y de moda y portadores como personas jóvenes.dinámicas, atléticas y atractivas (Fig. 15-20).

Figura 15: Leon

Figura 16: Finn 
Onomástica Desde América Latina, n.1, v.1, janeiro - junho, 2020, p. 77 -99. ISSN 2675-2719 https://doi.org/10.48075/odal.v1i1.24161

\section{Onogramm für Mia}

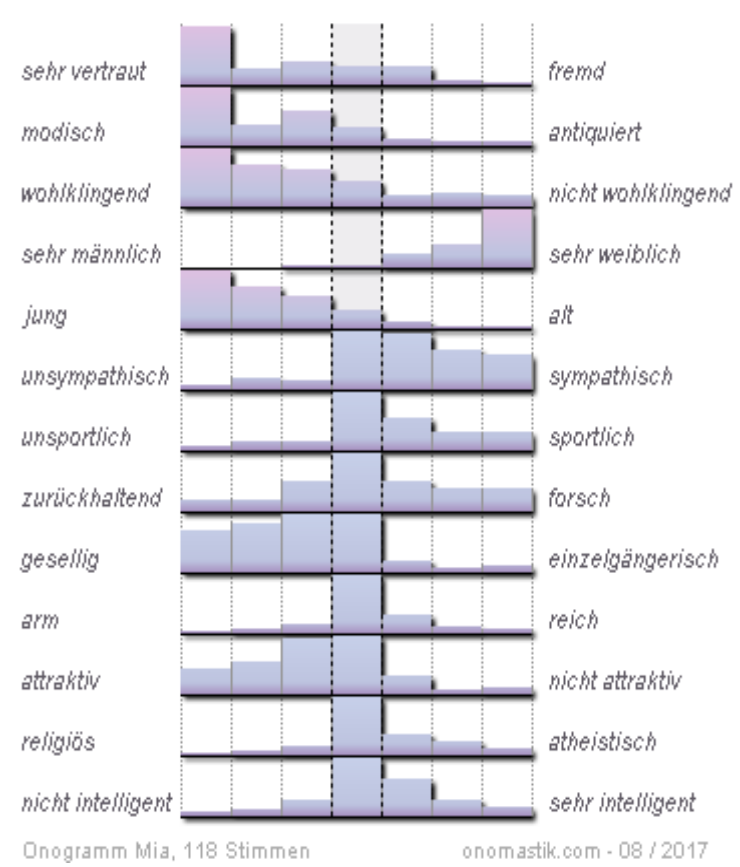

Figura 17: Mia

\section{Onogramm für Leni}

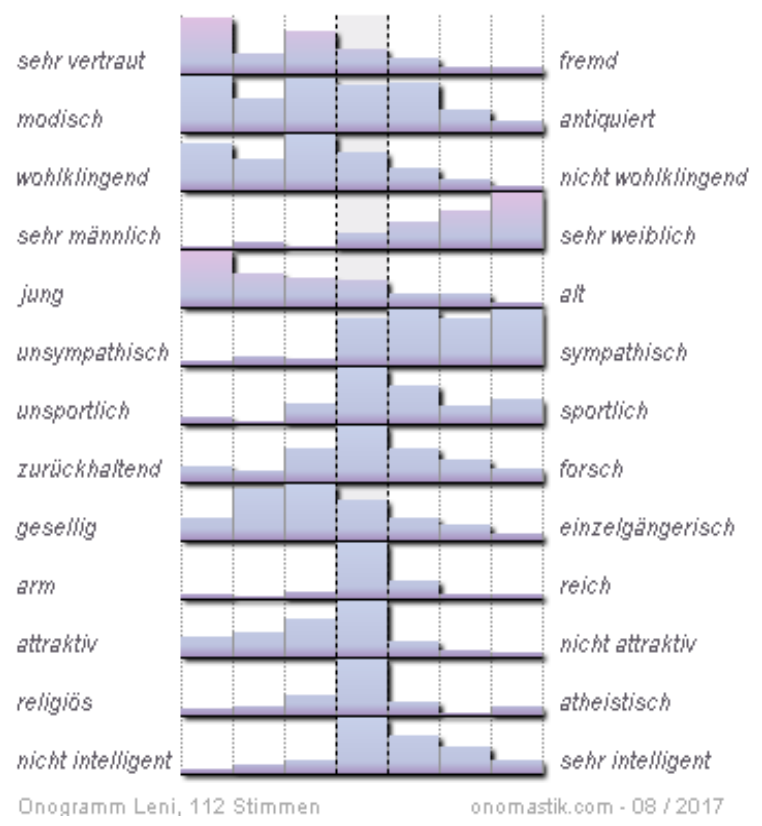

Figura 19: Leni

\section{Onogramm für Lilli}

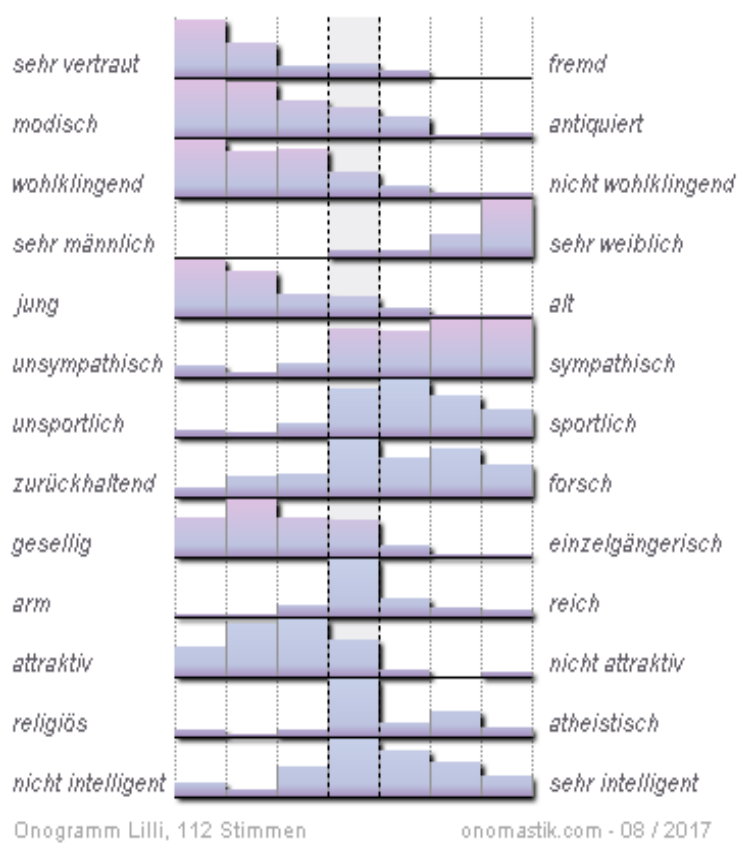

Figura 18: Lilli

\section{Onogramm ${ }^{\star}$ für Paul}

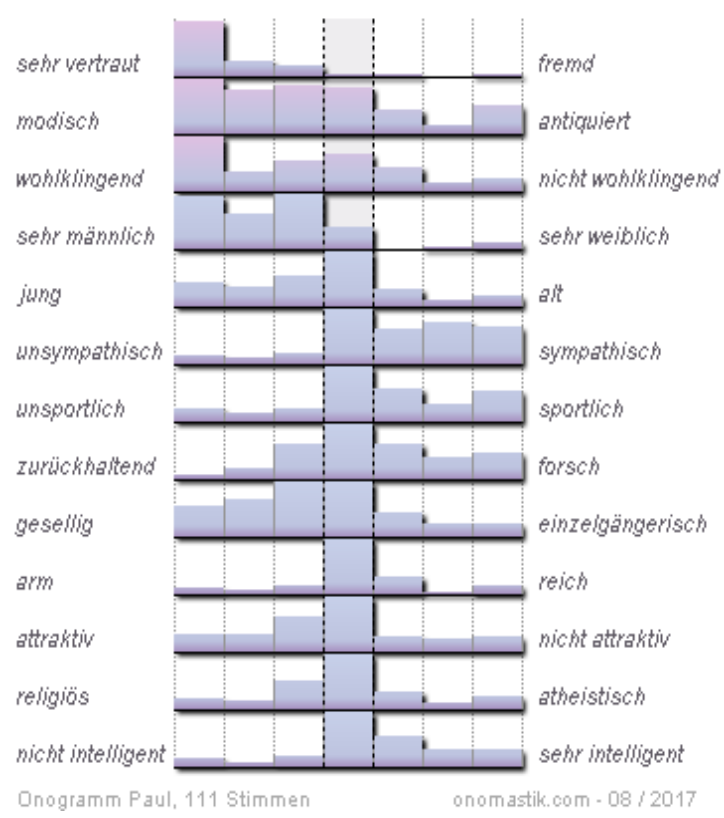

Figura 20: Paul 
Onomástica Desde América Latina, n.1, v.1, janeiro - junho, 2020, p. 77 -99. ISSN 2675-2719 https://doi.org/10.48075/odal.v1i1.24161

Los nombres de pila que fueron bastante populares en la RDA eran entre otros Mandy, Peggy y Silvio. Hoy se consideran más despectivamente y se percibe a las personas con estos nombres como menos inteligentes y menos atractivas.

Éste es el caso del nombre femenino Mandy, muy popular en la Ex-RDA (Figura 21). Según el estudio mencionado anteriormente (Kube, 2009) puede considerarse como el equivalente femenino del nombre Kevin. Los entrevistados en esa tesis de maestría respondieron que las niñas llamadas Mandy son de bajo rendimiento y con problemas de comportamiento. Como puede suponerse, en el este de Alemania hay numerosas mujeres y niñas con este nombre que no son ni de bajo rendimiento ni propensas a tener un mal comportamiento. Desafortunadamente, las percepciones de los habitantes del este de Alemania apenas fueron tomados en cuenta en el estudio antes mencionado. En consecuencia, las percepciones no son representativas de toda Alemania.

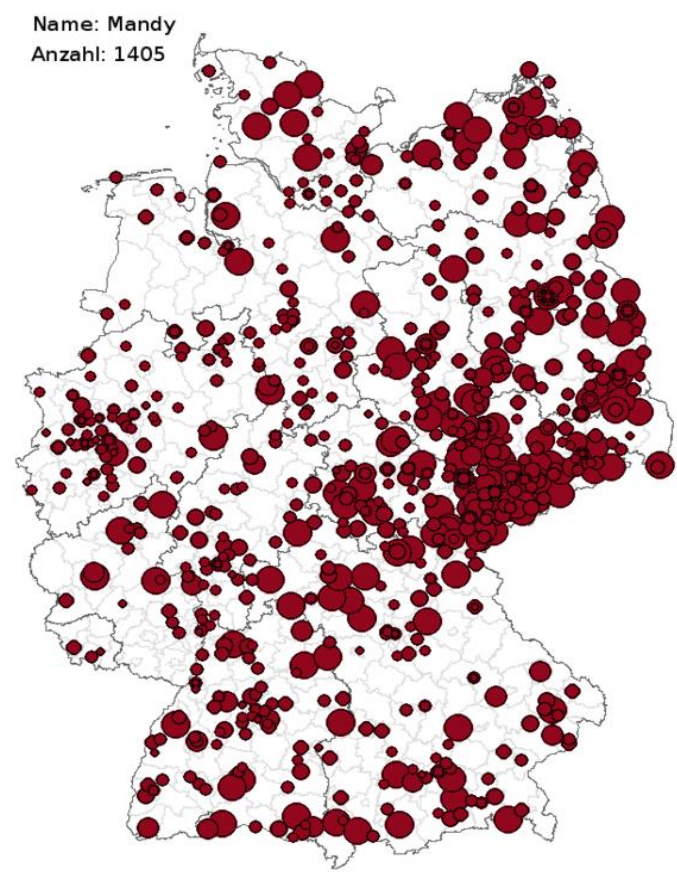

\section{Onogramm* für Mandy}

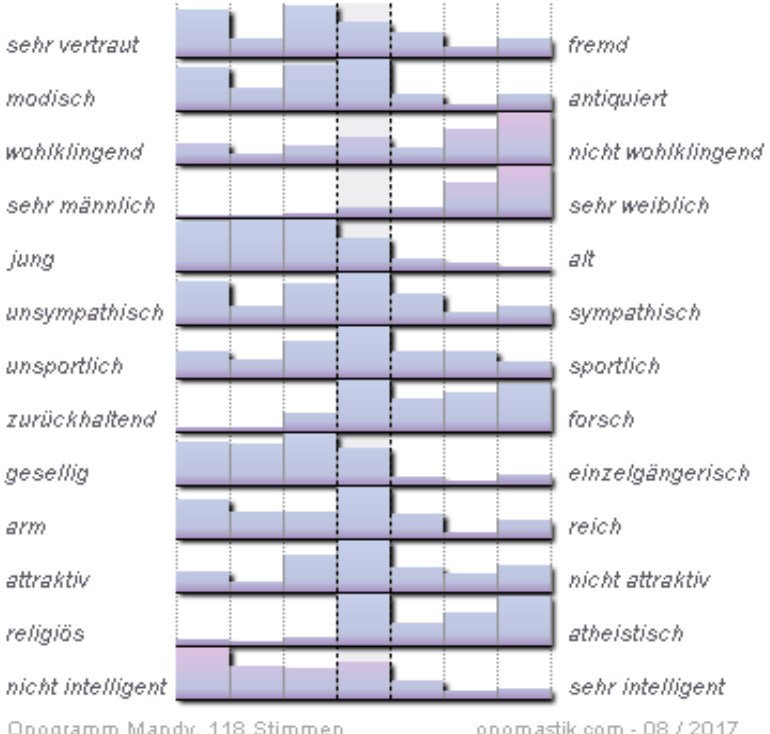

Figura 21: Mandy 
Onomástica Desde América Latina, n.1, v.1, janeiro - junho, 2020, p. 77 -99. ISSN 2675-2719 https://doi.org/10.48075/odal.v1i1.24161

Los onogramas para nombres de pila como Mandy, Peggy o Ronny confirman los prejuicios contra los alemanes orientales (Ex-RDA), que uno puede entrever detrás de estos nombres. En ellos se pueden observar los atributos dados a sus portadores: se los considera

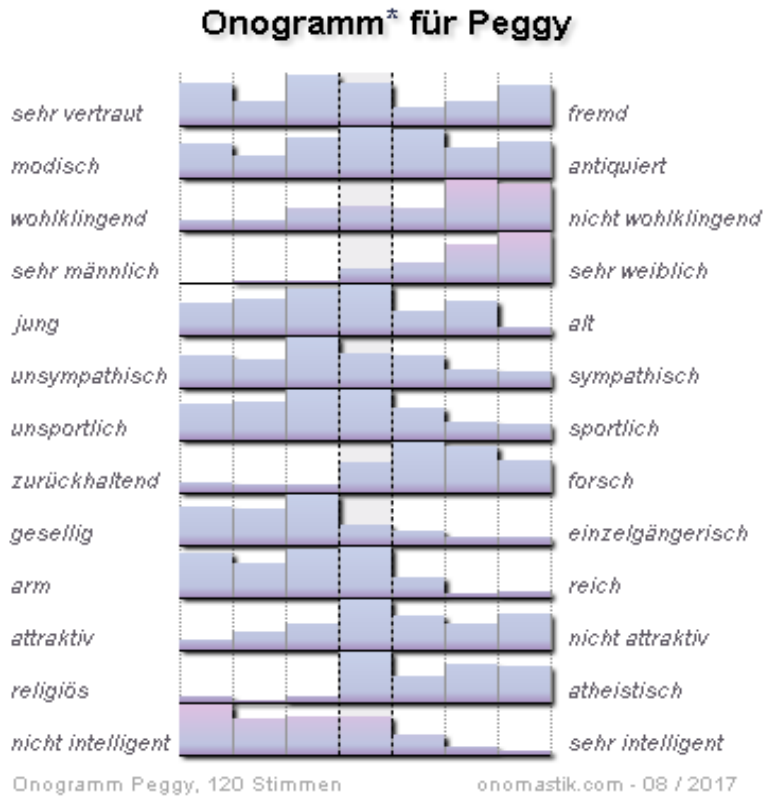


Onomástica Desde América Latina, n.1, v.1, janeiro - junho, 2020, p. 77 -99. ISSN 2675-2719 https://doi.org/10.48075/odal.v1i1.24161

como menos inteligentes, más pobres y menos atractivos (Figuras 22-24).

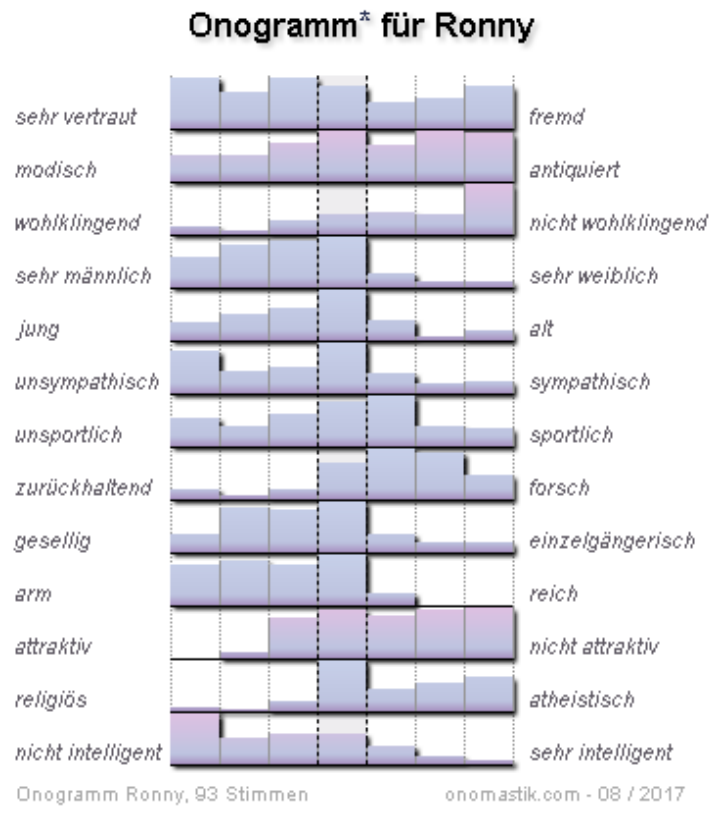

Figura 22 : Peggy; Figura 23: Ronny

De manera similar, los nombres extranjeros como Ali y Ais(c)ha, Aişe son percibidos en forma negativa. Estos nombres de pila son nuevos y no tienen tradición en Alemania. En consecuencia, son percibidos como extranjeros y más bien no melodiosos. Sus portadores se perciben como con mayor probabilidad de ser pobres, menos inteligentes y menos atractivos. Suelen asociarse al Islam y, en consecuencia, se los considera como muy religiosos (Figuras 24-25). 
Onomástica Desde América Latina, n.1, v.1, janeiro - junho, 2020, p. 77 -99. ISSN 2675-2719 https://doi.org/10.48075/odal.v1i1.24161

\section{Onogramm für Ali}

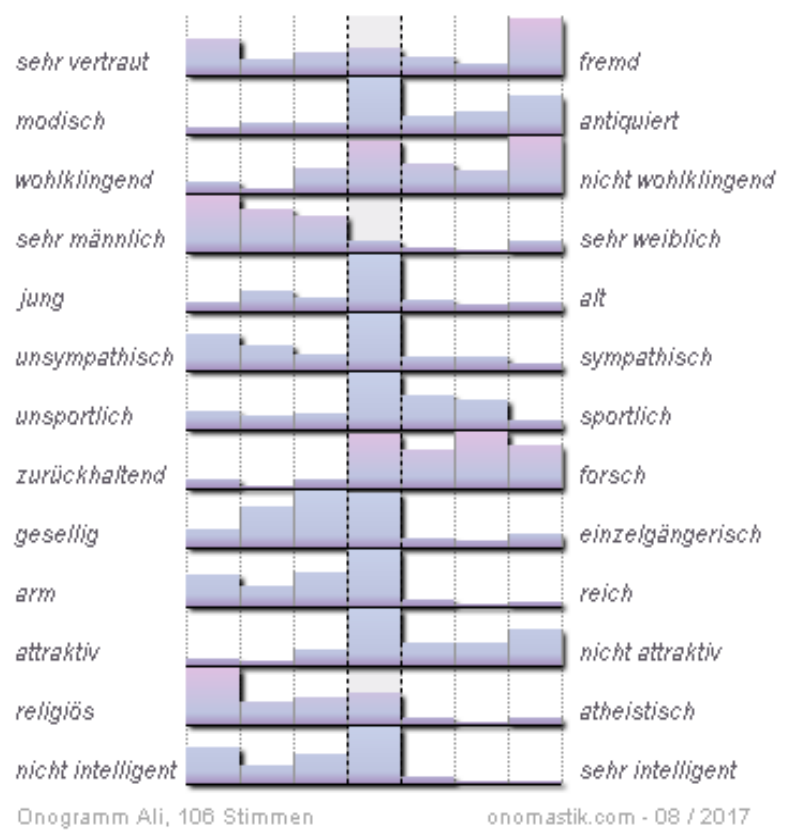

Figura 24: Ali
Onogramm für Aischa

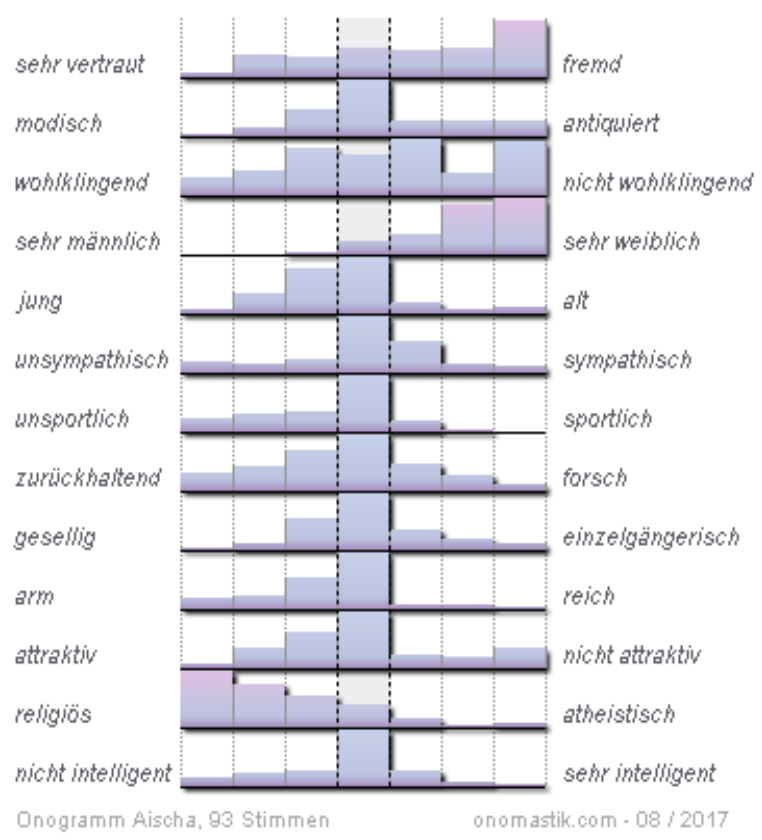

Figura 25: Aischa

Desde hace más de diez años se nota un renacimiento de los antiguos nombres de pila de origen alemán-germánico como Friedrich, Heinrich, Wilhelm, Bruno, Hugo, Richard, Frieda e Ida. Los onogramas de estos nombres a veces son muy equilibrados en cuanto a la edad y al atractivo de sus portadores (Figuras 26-30). 
Onomástica Desde América Latina, n.1, v.1, janeiro - junho, 2020, p. 77 -99. ISSN 2675-2719 https://doi.org/10.48075/odal.v1i1.24161

\section{Onogramm für Friedrich}

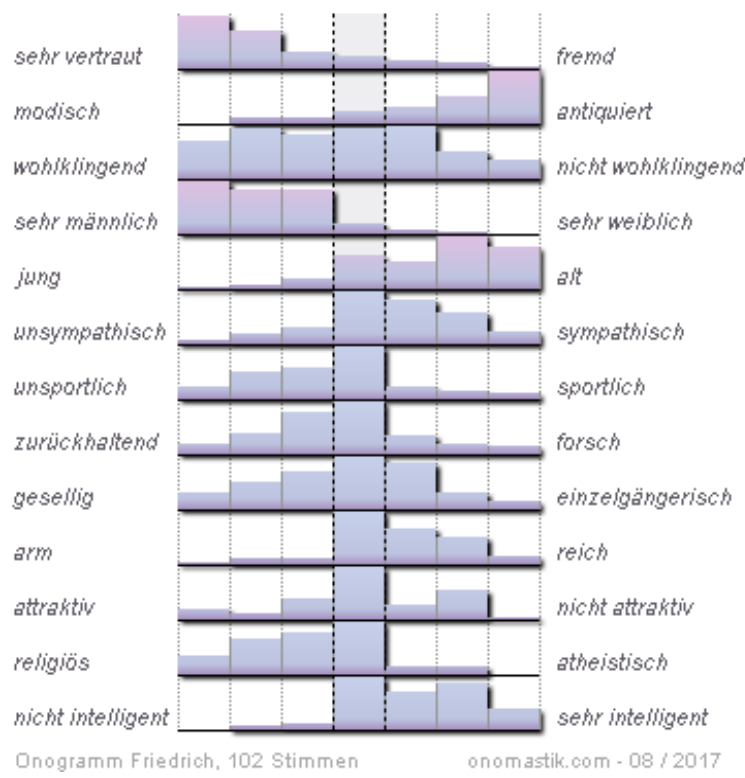

Figura 26: Friedrich

\section{Onogramm ${ }^{\star}$ für Frieda}

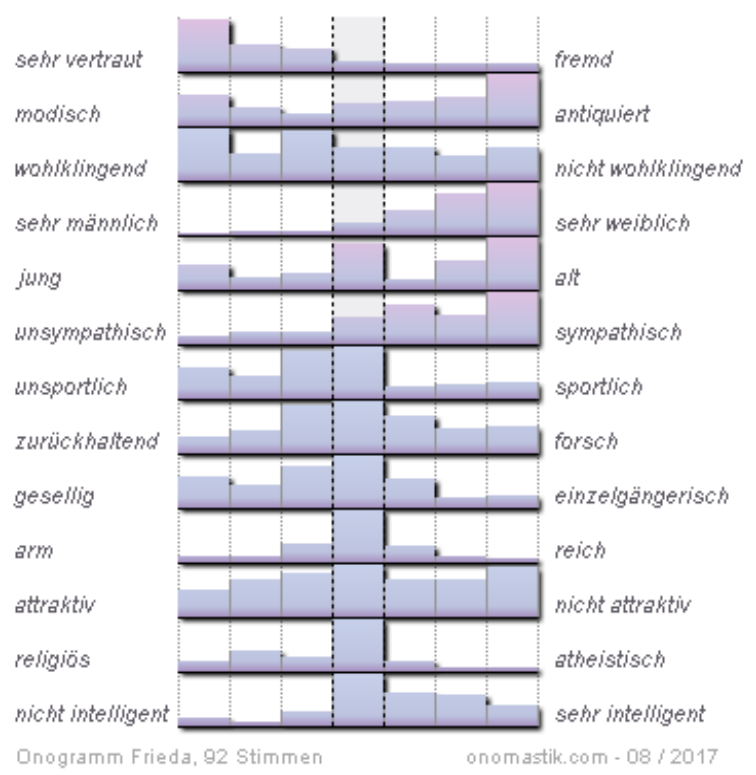

Figura 27: Frieda

\section{Onogramm für Karl}

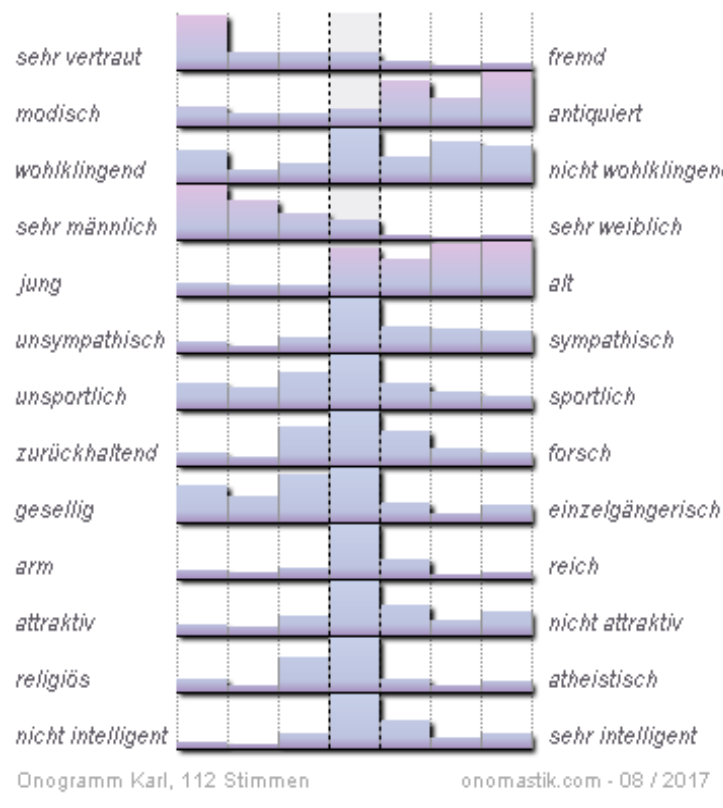

Figura 28: Karl

\section{Onogramm für Bruno}

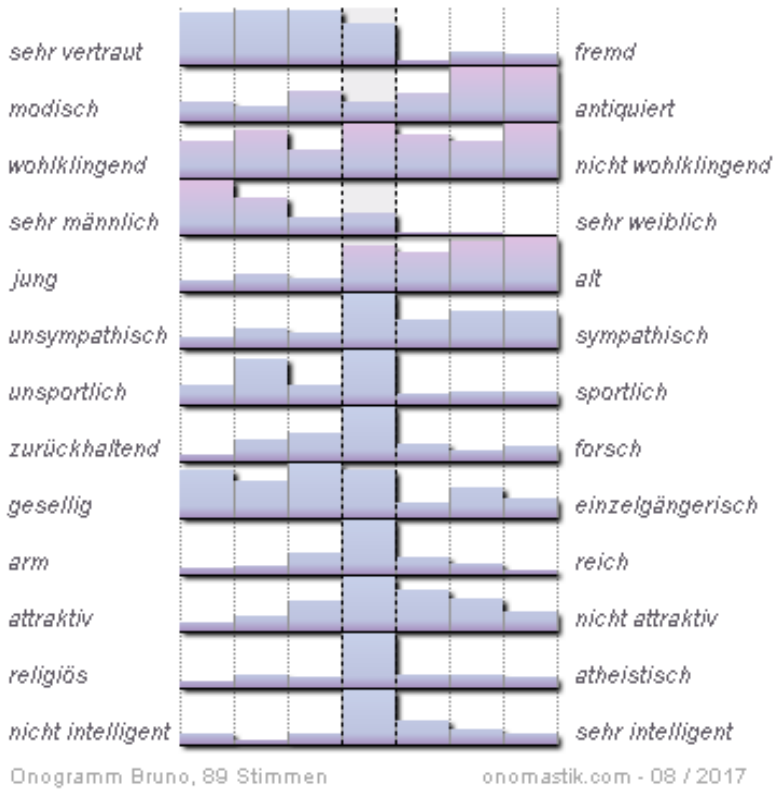

Figura 29: Bruno 
Onomástica Desde América Latina, n.1, v.1, janeiro - junho, 2020, p. 77 -99. ISSN 2675-2719 https://doi.org/10.48075/odal.v1i1.24161

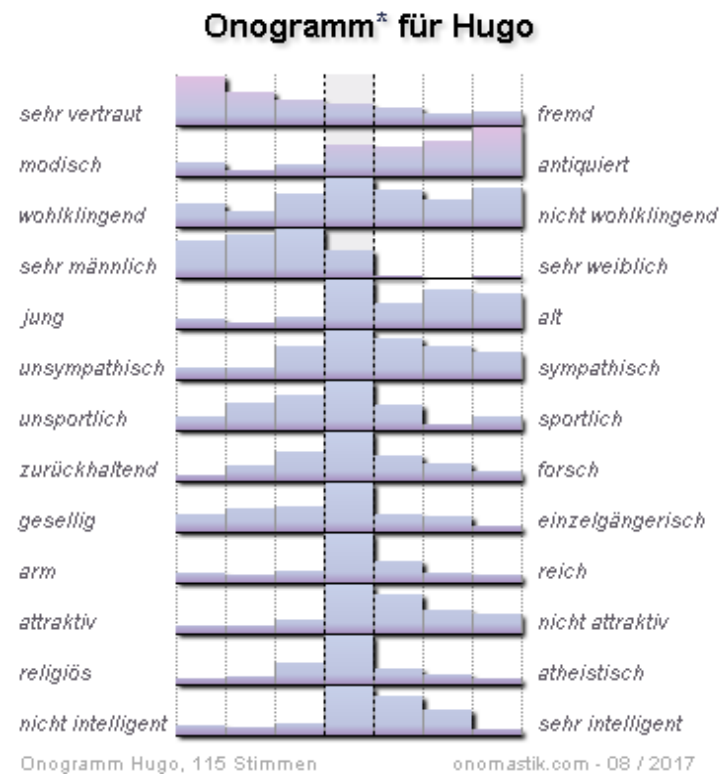

Figura 30: Hugo

El retorno al uso de estos nombres surgió hace más de diez años. Como parte del estudio a largo plazo Tendencias en los nombres de pila en Alemania, se realizó en el Centro de Onomástica de la Universidad de Leipzig entre 2006 y 2008 una encuesta en línea sobre diversos aspectos de la percepción de nombres de pila como, por ejemplo, la edad del portador del nombre.

Preguntamos por los nombres de pila Eberhard, Elfriede, Michael, Claudia, Friedrich, Paul, Frieda, Hugo, Bruno, Ida, Emma, Leni, Lilly y Maria, y por sus supuestos portadores. Como resultado de la evaluación de las respuestas, las personas llamadas Eberhard y Elfriede fueron consideradas al $100 \%$ como ancianos, mientras que las de nombre Michael y Claudia en un 30\%. Sin embargo, los nombres Michael y Claudia no se percibieron como nombres modernos. Los nombres bien conocidos y hoy populares, como Friedrich, Paul, Frieda, Hugo, Bruno, Ida, Emma, Leni, Lilly y Maria todavía en 20062008 fueron evaluados como nombres antiguos, aunque a veces ya como nombres modernos. 
Onomástica Desde América Latina, n.1, v.1, janeiro - junho, 2020, p. 77 -99. ISSN 2675-2719 https://doi.org/10.48075/odal.v1i1.24161

Es interesante hacer notar que las personas llamadas Maria y Michael fueron consideradas muy religiosas: Maria incluso en un $80 \%$. Aquí los conocimientos culturales de quien participaba en la encuesta acerca de la Virgen María y el arcángel San Miguel parecieran sesgar la evaluación real de los portadores del nombre.

\begin{tabular}{|l|l|l|}
\hline Nombre & Antiguo & Moderno \\
\hline Eberhard / Elfriede & $100 \%$ & \\
\hline Claudia / Michael & $30 \%$ & \\
\hline Friedrich & $73 \%$ & $16 \%$ \\
\hline Paul & $43 \%$ & $48 \%$ \\
\hline Frieda & $83 \%$, & $7 \%$ \\
\hline Hugo / Bruno & $71 \%$ & $10 \%$ \\
\hline Ida & $53 \%$ & $10 \%$ \\
\hline Emma & $41 \%$ & $28 \%$ \\
\hline Leni & $23 \%$ & $38 \%$ \\
\hline Lilly & $13 \%$ & $55 \%$ \\
\hline Maria & $38 \%$ & $31 \%$ \\
\hline
\end{tabular}

Tabla Resultados parciales de la encuesta en línea "Percepción de los nombres" del Centro de Onomástica de la Universidad de Leipzig, durante los años 2006-2008.

En los perfiles de impacto gráfico (onogramas) las percepciones actuales de la edad de los nombres de pila o de los portadores de nombres están obviamente claramente definidas.No se puede decir, sin embargo, cómo es la autenticidad de los resultados de los otros aspectos: el atractivo, la religiosidad, la riqueza, la pobreza y la inteligencia.

Cómo se puede ver que los portadores de los nombres Maria, Michael o Gabriele se ven como personas muy religiosas, es más probable que se refiera a la información sobre los nombres y su historia y menos sobre los nombres actuales. Se debe suponer una correlación eufonía y atractivo, así como la edad y la deportividad.

Este trabajo es solo una pequeña parte de una investigación amplia a larga plazo. Se puede ver por ejemplo, que los nombres germánicos, de eran moda a principios del siglo $\mathrm{XX}$, regresan ahora, al cabo de 4 generaciones, lo que coincide con otros estudios realizados internacionalmente. Como, mediante los onogramas, se puede mostrar con 
Onomástica Desde América Latina, n.1, v.1, janeiro - junho, 2020, p. 77 -99. ISSN 2675-2719 https://doi.org/10.48075/odal.v1i1.24161

claridad la percepción que los hablantes tienen de los nombres de pila en uso y de sus portadores, eso pone en evidencia los posibles prejuicios (positivos y negativos) hacia ciertos grupos de personas de la población actual en Alemania.

Una desventaja de los onogramas es que la encuesta se realiza de forma anónima. Por lo tanto, no hay ninguna información sobre la edad, el origen y el nivel educativo de los encuestados. En consecuencia, tenemos analizar los resultados en sus tendencias. Queda por ver si los cambios en la valoración pueden detectarse durante un período de tiempo más largo.

Recebido em 21/02/2020 Aceito em 26/02/2020 Publicado em 03/03/2020

\section{Referencias}

Datenbank der Namenberatung der Universität Leipzig.

Hartmann T. (1984) Untersuchung der konnotativen Bedeutung von Personennamen. Ein theoretischer und empirischer Beitrag zur Psychoonomastik mit Hilfe eines konzeptspezifischen semantischen Differentials. Neumünster: Wachholz.

https://www.onomastik.com/Vornamen-Lexikon/

https://www.welt.de/News/article104680997/Kevin-Das-ist-kein-Name-sondern-eine-

Diagnose.html. (diciembre 2017)

https://de.wikipedia.org/wiki/Kevin. (diciembre 2017)

http://de.uncyclopedia.wikia.com/wiki/Kevinismus (diciembre 2017).

Krien R. (1973) Namenphysiognomik: Untersuchungen zur sprachlichen Expressivität am Beispiel von Personennamen, Appellativen und Phonemen des Deutschen. Tübingen: Max Niemeyer Verlag.

Kube J. I. (2009) Vornamensforschung, Fragebogenuntersuchung bei Lehrerinnen und Lehrern, ob Vorurteile bezüglich spezifischer Vornamen von Grundschülern und davon abgeleitete erwartete spezifische Persönlichkeitsmerkmale vorliegen, Hochschulschrift zugl.: Oldenburg, Univ., Master-Arbeit. 
Onomástica Desde América Latina, n.1, v.1, janeiro - junho, 2020, p. 77 -99. ISSN 2675-2719 https://doi.org/10.48075/odal.v1i1.24161

Rudolph U., Böhm R. und Lummer M. (2007) Ein Vorname sagt mehr als 1.000 Worte. Zur sozialen Wahrnehmung von Vornamen. Zeitschrift für Sozialpsychologie Dezember 2006 38(1):17-31.

Utech U. (2011) Rufnamen und soziale Herkunft. Studien zur schichtenspezifischen Vornamenvergabe in Deutschland. Hildesheim - Zürich- NY: Georg Olms Verlag. Germanistische Linguistik Monographien, Band 25. 